\title{
A CMOS rail-to-rail linear VI-converter
}

\author{
P. P. Vervoort and R. F. Wassenaar \\ MESA Research Institute \\ Twente University \\ P.O. Box 217 \\ 7500 AE Enschede, the Netherlands \\ Phone: $x-3153892732$ \\ Fax: x-31 53341903
}

\begin{abstract}
A linear CMOS VI-converter operating in strong inversion with a commonmode input range from the negative to the positive supply rail is presented. The circuit consists of three linear VI-converters based on the difference of squares principle [1-3]. Two of these perform the actual $V$ to $I$ conversion, while the third changes the bias currents of the first two in response to changes in the input common-mode level.
\end{abstract}

The resulting circuit has a large signal transconductance which is constant to within $3 \%$ over the entire common-mode input range. It can operate from a single supply voltage of 2.2Volts.

\section{Introduction}

The common-mode input range of a single $(\mathrm{N}$ type) transconductor reaches from a certain voltage level above the negative supply rail up to a certain level above the positive supply rail. In order to cover the entire rail-to-rail commonmode input range, an N-type VI-converter and a complementary P-type have to be driven in parallel. A special circuit is needed to combine the output currents of the individual VIconverters. The combined large signal transconductance can be made independent of the common-mode input level by biasing the $\mathrm{N}$-type and P-type VI-converters with the individual output currents of a third VI-converter after current limiting. This third VI-converter is singleended and driven by the common-mode input voltage. Figure 1 shows the structure of the proposed VI-converter circuit.

\section{Principle}

The individual output currents of a linear VIconverter based on the difference of squares principle can be described as:

$$
\begin{gathered}
I_{d 1}=\frac{K}{2}\left(V_{d c}+V_{i n}\right)^{2} \text { and } I_{d 2}=\frac{K}{2}\left(V_{d c}-V_{i n}\right)^{2} \quad(I) \\
\text { with } K=\mu C_{o x} \frac{W}{L}
\end{gathered}
$$

and therefore:

$$
I_{d 1}-I_{d 2}=g_{m} V_{\text {in }} \text { with } g_{m}=2 K V_{d c}
$$

If the DC-voltage source $V_{d c}$ is formed by the $V_{g s}$ of a MOSFET with a constant drain current $I_{d c}$ the large signal transconductance value of the VIconverter is proportional to $\sqrt{K I_{d c}}$. If the

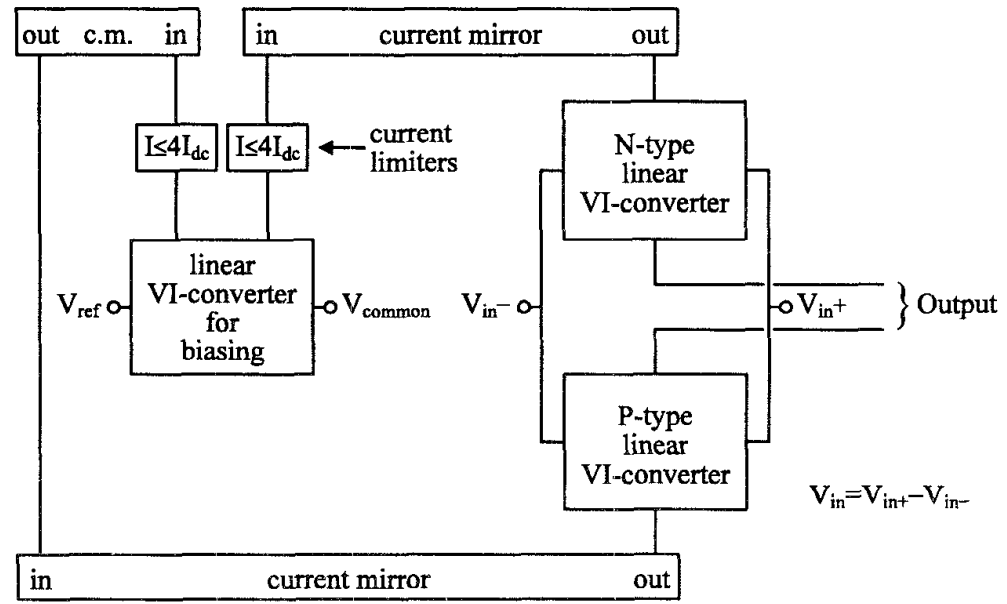

Fig. 1 Structure of the VI-converter circuit. 
total $g_{m}$ of the circuit of figure 1 has to be constant, then the condition:

$$
g_{m N}+g_{m P}=\text { constant }
$$

has to be satisfied. Since $g_{m}$ is proportional to $\sqrt{K I_{d c}}$ and assuming $K_{N}=K_{P}$ equation 3 can be rewritten as:

$$
\sqrt{I_{d c N}}+\sqrt{I_{d c P}}=\text { constant }
$$

It now happens that the currents of equation 1 satisfy this condition:

$$
\sqrt{I_{d 1}}+\sqrt{I_{d 2}}=2 \sqrt{\frac{K}{2}} V_{d c}
$$

if $V_{d c}$ is constant. This means that these currents can be provided by a similar VI-converter.

If one of both currents becomes zero, the other one has to be limited to a constant value in order to keep the total transconductance constant, this explains the need for the current limiters in figure 1 .

\section{Circuit description}

A possible realisation of a transconductor based on the difference of squares principle is the circuit shown in figure 2 . Here the DC-voltage sources are formed by MOSFET's M3 and M4. Their drain currents are kept constant by the current sources M5 and M6 and the feedback loops formed by M7, M9 and M8, M10. The transistors $\mathrm{M} 7$ and $\mathrm{M} 8$ disconnect the drains of M3 and M4 from the gates of M9 and M10 and so enlarge the common-mode input range. This transconductor has been selected for its low minimal supply voltage and its efficient use of current.

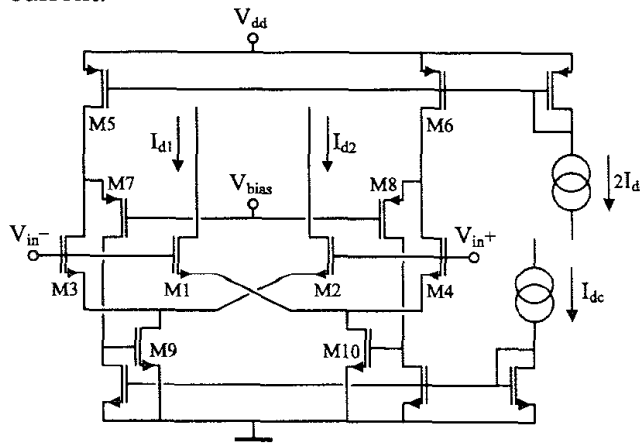

Fig. 2 Schematic of a square-law transconductor

After some modification the transconductor of figure 2 can also be used as the biasing circuit. In figure 3 , one half of the circuit of the transconductor of figure 2 is shown together with a circuit that limits its output current to a value of $4 I_{d c}$. The current limiting is based on the principle that in a series connection of a current sink and a current source the lowest current always dominates [4]. The transistor that wants to carry the larger current is forced into the triode region in order to carry the lower current. In figure 3 the output of the current mirror formed by M11-M14 act as the current source and is connected in series with the drain of transistor M1 which acts as the current sink. A mirrored copy of the drain currents of M1, M13 and M14 is available at the drain of transistor M15.

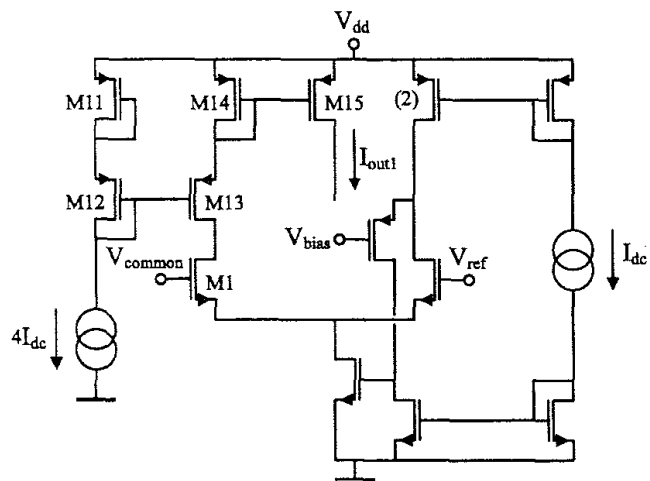

Fig. 3 Part of the transconductor with current limiter.

A combination of the circuit of figure 3 and its complementary version can be used to bias the two linear transconductors as is shown in figure 4. The complementary version of the circuit of figure 3 is used in order to eliminate the need for one of the current mirrors in figure 1 . The input voltages for the biasing circuit will be a reference voltage (normally $V_{d d} / 2$ ) and the common-mode input voltage.

The transconductor of figure 2 and its complementary version are used for the transconductors of figure 1. The transconductor with the N-type input transistors (like the one in figure 2) is biased as a function of the commonmode input level by using the circuit of figure 3 . The complementary version of the transconductor (with P-type input transistors) is biased by a complementary version of the circuit of figure 3 . This results in the complete linear transconductor with a rail-to-rail common-mode input range, shown in figure 4. 


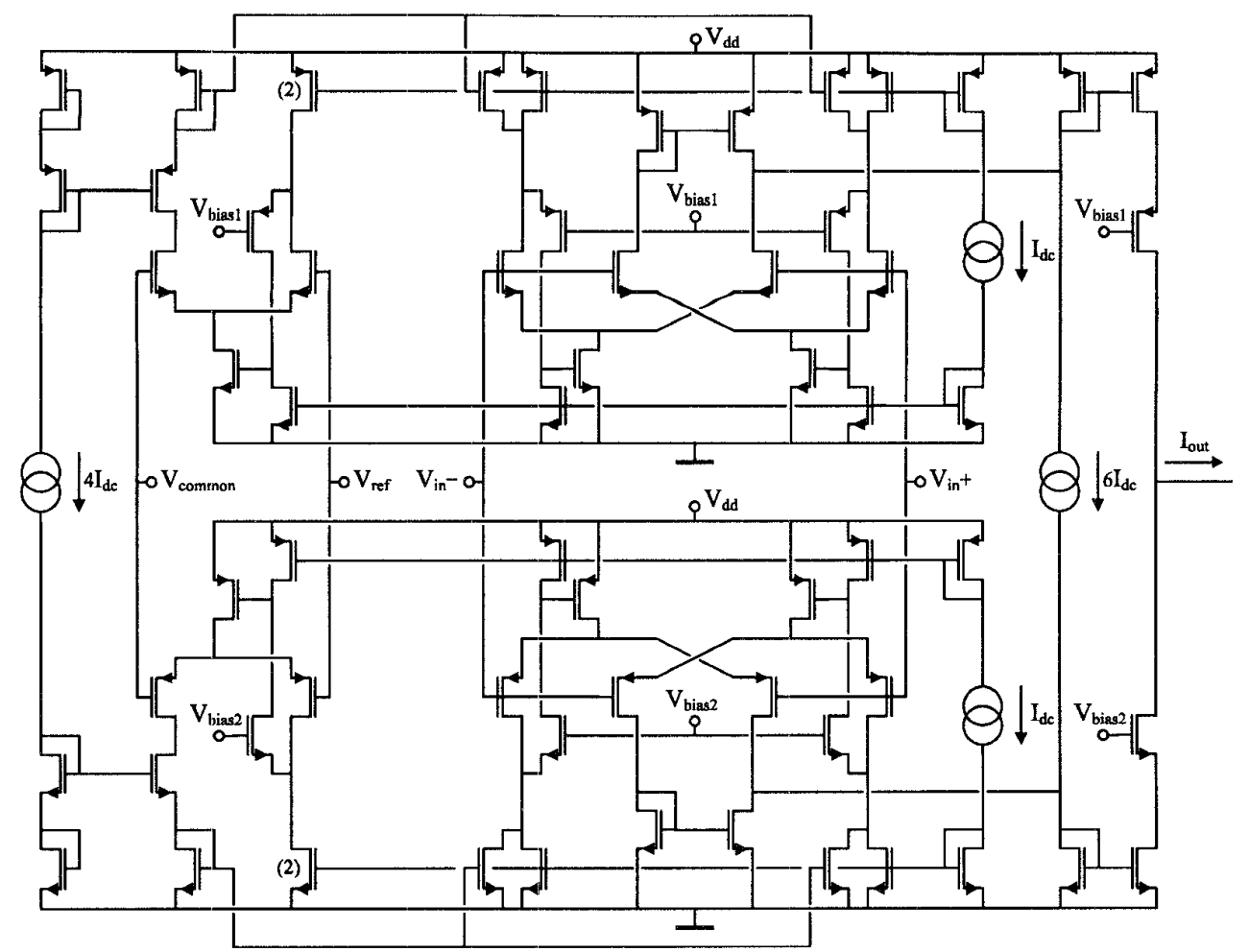

Fig. 4 Complete circuit of the linear VI-converter.

\section{Simulations:}

Several PSPICE simulations have been performed with the circuit of figure 4 , using the parameters of a $2.5 \mu \mathrm{m}$ CMOS process and a supply-voltage of 3 Volts. The value of $I_{d c}$ was $8 \mu \mathrm{A}$. The length of the transistors used for the actual VI-converters equals $10 \mu \mathrm{m}$. The width of the PMOS transistors was taken 3.2 times the width of the NMOS transistors in an attempt to make $K_{P}$ equal $K_{N}$. Under these conditions a small-signal bandwidth of $4.8 \mathrm{MHz}$ can be achieved. The adaptive biasing circuit reduces the variation of the transconductance from $33 \%$ to less than $3 \%$. Using an $I_{d c}$ of $2 \mu \mathrm{A}$ the minimal supply voltage is 2.2 Volts.

Table 1 gives an overview of several performance characteristics of the presented circuit. The figures are given for a nominal value for $V_{\text {common }}$ which is equal to $V_{d d} / 2$ and the value over the entire common-mode input range ( $V_{\text {common }}$ varies between 0 to $V_{d d}$ ).

\begin{tabular}{|c|c|c|c|}
\hline & $\begin{array}{l}\text { nominal } \\
\text { value }\end{array}$ & $\begin{array}{l}\text { over entire common- } \\
\text { mode range }\end{array}$ & \\
\hline DC-transconductance & 116 & $110-116$ & $\mu \mathrm{A} / \mathrm{V}$ \\
\hline Small signal bandwidth (-3dB point) & 4.8 & $4.8-9.0$ & $\mathrm{MHz}$ \\
\hline Equivalent input noise & 47 & $45-48$ & $\mathrm{nV} / \sqrt{\mathrm{Hz}}$ \\
\hline $\begin{array}{l}\text { THD differential input voltage } \\
\left(\mathrm{V}_{\mathrm{in}}=400 \mathrm{mV}_{\mathrm{pD}}\right)\end{array}$ & 0.14 & $0.14-1.4$ & $\%$ \\
\hline $\begin{array}{l}\text { THD single-ended input voltage } \\
\left(\mathrm{V}_{\text {in }}=400 \mathrm{mV}_{\mathrm{pp}}\right)\end{array}$ & 0.4 & $0.04-1$ & $\%$ \\
\hline $\begin{array}{l}\text { CMRR } \\
V_{\text {common }}=V_{\text {common dc }}+V_{a c}\left(100 \mathrm{mV}_{\mathrm{tt}}, 100 \mathrm{~Hz}\right)\end{array}$ & 58 & $26-58$ & $\mathrm{~dB}$ \\
\hline $\begin{array}{l}\text { PSRR } \\
V_{d d}=3 V_{d c}+V_{a c}\left(100 m V_{t t}, 100 H z\right)\end{array}$ & 48 & $25-49$ & $\mathrm{~dB}$ \\
\hline Supply current & 300 & $300-400$ & $\mu \mathrm{A}$ \\
\hline
\end{tabular}

Table 1 Simulated performance characteristics of the transconductor. 


\section{Conclusions}

A low-voltage linear CMOS transconductor with a rail-to-rail common-mode input range and a large signal transconductance which is independent of the common-mode input voltage has been presented. The circuit can be used for differential signals with varying common-mode levels or single-ended signals. In both cases the transconductance value will remain constant within $3 \%$. It can operate on a supply voltage of 2.2 Volts or higher.

\section{Acknowledgements}

The authors would like to thank K. Bult and C.J. Abel for their assistance during this project.

\section{References}

[1] Nedungadi, A. P., Viswanathan, T. R.: "Design of linear CMOS transconductance elements", IEEE Transistor Circuits and Systems, 1984, CAS-31, pp. 891-894

[2] Seevinck, E., Wassenaar, R. F.: "A versatile CMOS linear transconductor/square-law function circuit", IEEE J. solid-state circuits, 1987, Vol. SC-22, pp.366-377

[3] Viswanathan, T. R.: "CMOS transconductance element", Proceedings of the IEEE, 1986, Vol. 74, pp. 222-224

[4] Botma, J. H., Wassenaar, R. F., Wiegerink, R. J.: "Simple rail-to-rail lowvoltage constant-transconductance CMOS input stage in weak inversion", Electron. Lett., 1993, Vol. 29, no. 12, pp.1145-1146 